A. Pramesh Rao, G. Swarup and Gopal-Krishna, eds.

\title{
Atomic Hydrogen Gas Images of QSO Host Galaxies
}

\author{
J. Lim \\ Academia Sinica Institute of Astronomy \& Astrophysics, PO Box 1-87, \\ Nankang, Taipei 115, Taiwan \\ P.T.P. Ho \\ Smithsonian Astrophysical Observatory, 60 Garden Street, Cambridge, \\ MA 02138, USA
}

\begin{abstract}
We present the first neutral atomic hydrogen (HI) gas images of QSO host galaxies. The results come from our ongoing survey of all twenty-three QSOs at redshifts $z<0.07$ visible from the VLA. Our images show tidal interactions in all of the QSO host galaxies detected in HI, even when no such interactions are clearly visible in the optical. The results are not consistent with the suggestion by Sanders et al. (1988a, 1988b) that ultraluminous infrared galaxies, the vast majority of which are later-stage mergers, are the parent population of a significant fraction of radio-quiet QSOs. Instead, our results suggest that QSOs reside in a wide variety of interacting environments.
\end{abstract}

\section{Introduction}

Quasi-Stellar Objects (QSOs) are generally believed to be the optically visible evidence for the vigorous accretion of gas onto supermassive black holes at the centers of host galaxies. They first appear in great numbers at high redshifts of $z \geq 2-3$, and below redshifts of $z \approx 1-2$ decrease dramatically in both comoving number density as well as peak luminosity. Their genesis and observed cosmological evolution constitute one of the outstanding mysteries of modern astronomy.

There are two widely-debated models for the origin of QSOs. In the Pure Luminosity Evolution (PLE) model, a single generation of QSOs were born at high redshifts and have since evolved mainly by decreasing in luminosity. In this model, lower redshift QSOs are the descendents of their much more luminous counterparts at high redshifts, and ought to reside in otherwise passively evolving (massive) host galaxies. By contrast, in the Pure Density Evolution $(P D E)$ model, successive generations of QSOs are born at different redshifts, and conspire to produce the observed cosmological evolution. In this model, the mechanism most frequently cited to trigger QSO activity is interactions and mergers involving at least one gas-rich galaxy, and hence QSOs ought to reside in violently interacting or merging systems of galaxies.

Although optical images of QSO host galaxies have improved dramatically over the past several decades, rigorous proof for either of the above scenar- 
ios remains elusive. Ground-based optical imaging reveal that a fraction of low-redshift QSO host galaxies display morphological peculiarities attributed to gravitational interactions between galaxies, and that a large fraction are accompanied by projected neighboring galaxies cited as circumstantial evidence for galactic interactions (e.g, Hutchings \& Campbell 1983; Hutchings, Crampton, \& Campbell 1984; Smith et al. 1986; Yee 1987). The clearest optical images of QSO host galaxies, however, come from space-based observations with the Hubble Space Telescope (HST). In a study of thirty-four QSOs at redshifts $z \approx 0.1-0.5$ (Bahcall et al. 1997; Boyce et al. 1998); the majority of which have previously been imaged from the ground, only six were found to lie in complex interacting systems; the remainder reside in apparently normal disk and elliptical galaxies, although two-thirds have projected neighboring galaxies. A central conclusion of these studies is that QSOs reside in a broad range of environments.

Here, we show the first images of QSO host galaxies taken in neutral atomic hydrogen (HI) gas. In nearby galaxies, $\mathrm{HI}$ is known to be an exquisite tracer of tidal interactions (e.g., review of Giovanelli \& Haynes 1988), even when none are readily visible in the optical. This is because in normal disk galaxies, HI extends further out than the stellar disk, where it is more susceptible to disruptions by external gravitational forces; thus being more widely dispersed, HI also takes longer to gravitationally relax. The contrasting ability of optical and HI images to reveal gravitational interactions between galaxies is beautifully demonstrated by the study of the M81 group of galaxies. This system, which contains the nearest starburst galaxy M82, shows no obvious evidence for interactions in the optical. By contrast, the HI image published by Yun, Ho, \& Lo (1996) shows filamentary structures connecting different galaxies in the group, clearly delineating tidal interactions between these galaxies. Apart from revealing any tidal interactions, HI observations of QSO host galaxies also are important for studying the spatial and kinematic structure of their neutral atomic gas component.

\section{Observations and Results}

We are conducting an ongoing $\mathrm{HI}$ imaging survey of all twenty-three QSOs with redshifts $z<0.07$, as listed in the catalog of Veron-Cetty \& Veron (1998), visible from the Very Large Array (VLA). The vast majority of these objects are radio-quiet QSOs. In Figure 1, we show the results for six of the objects in our sample, arranged in order of decreasing projected linear separation between the QSO and (any) interacting neighboring galaxy. Three of these images have previously been reported in Lim \& Ho (1999).

In all cases where the QSO host galaxy was detected, the HI gas shows disruptions consistent with gravitational interactions with (any) neigboring galaxies as indicated in the individual images. This disruption often takes the form of tidal bridges extending between the interacting galaxies, and/or tidal tails extending away from the opposing interacting galaxy. There is only one example of a likely merger remnant, the spheroidal host galaxy of Markarian 304, which has no detectable (either in the optical or $\mathrm{HI}$ ) neighboring galaxies, and which shows what seem to be two tidal tails. In the case of $4 U 0241+61$, whose host galaxy was not detected in $\mathrm{HI}$, the $\mathrm{HI}$ image reveals a neighboring galaxy with an apparent tidal bridge on the side of the QSO host galaxy. 

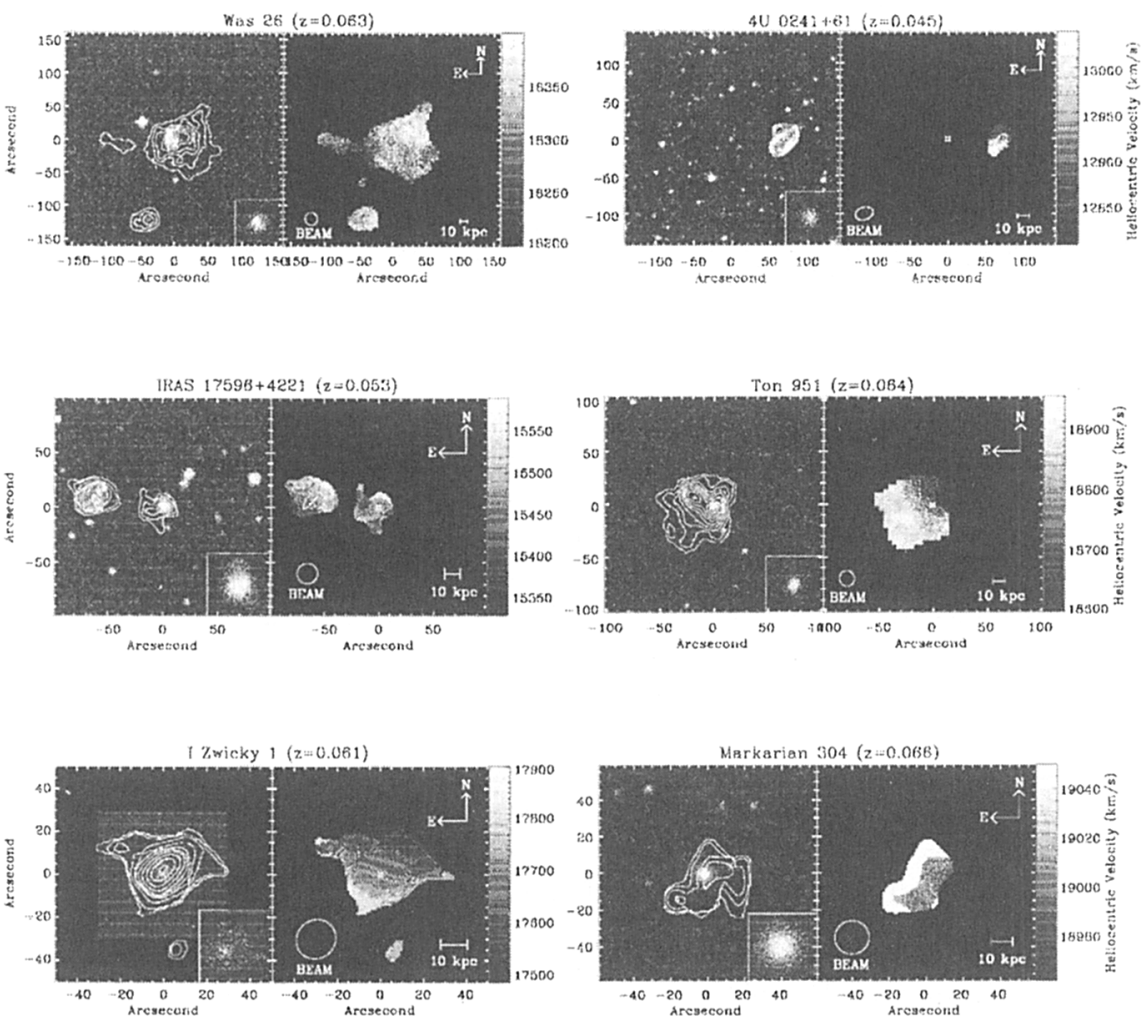

Figure 1. Selected results from our HI imaging survey of QSO host galaxies, arranged in sequence of decreasing projected linear separation between the QSO and (any) likely neighboring interacting galaxies. Left-hand panels show contour plots of the HI gas distribution (integrated intensity) superposed on grayscale optical images, and righthand panels show grayscale plots of the HI gas kinematics (intensity weighted mean velocity) with velocity scales indicated by the adjacent vertical wedges. The QSO is at the center of each image, and is shown magnified in the lower right inset of each left-hand panel. In each righthand panel, the QSO is marked by a star symbol, while any neighboring galaxies is marked by a circle or ellipse. 


\section{Discussion and Conclusion}

Our HI images reveal tidal interactions in all of the detectable QSO host galaxies, even when no such interactions are readily if at all apparent in the optical. Such disruptions in the HI gas can also be seen when there are no apparent neighboring galaxies, presumably indicative of a merger.

Sanders et al. (1988a, 1988b) have suggested that ultraluminous infrared galaxies, nearly all of which appear to be late-stage mergers, are the parent population of at least a significant fraction of radio-quiet QSOs. In this scenario, nuclear activity is activated after the infrared-luminous starburst phase when sufficient gas reaches the center of the galaxy to vigorously fuel the central supermssive black hole. Furthermore, the QSO may only become visible (in the optical) long only after the starburst phase, when the surrounding dust has had time to disperse. By this time, the galaxy may have completely merged and become largely relaxed in the optical. Our results, however, are not consistent with this simple picture. There is only one example of a likely merged remnant, Markarian 304. In at least half of the cases shown in Figure 1, the interacting systems have relatively simple linear tidal features characteristic of a relatively early phase of an interaction (e.g., $4 \mathrm{U} 0241+61$, IRAS $17596+4221$, and perhaps also I Zwicky 1) and/or large projected separations with its interacting neighboring galaxy (e.g., Was 26). Instead, our results suggest that radio-quiet QSOs can be found in a wide variety of interacting environments.

Acknowledgments. This project is supported in part by a grant from the National Science Council (NSC) of Taiwan to J. Lim. He would like to thank the NSC for financial support to attend this symposium. We also would like to thank two undergraduate students from National Taiwan University (NTU), Chuo Hua-Ting and Wen Shyang, who helped in the data reduction for some of the images shown in Figure 1.

\section{References}

Bahcall, J. N., Kirhakos, S., Saxe, D. H., \& Schneider, D. P. 1997, ApJ, 479, 642

Boyce, P. J., Disney, M. J., Blades, J. C., Boksenberg, A., Crane, P., Deharveng, J. M., Macchetto, F. D., Mackay, C. D., \& Sparks, W. B. 1998, MNRAS, 298,121

Giovanelli, R. \& Haynes, M. P. 1988, in Galactic and Extragalactic Radio Astronomy, ed. G. L. Verschuur \& K. I. Kellermann, (Springer-Verlag), 522

Hutchings, J. B. \& Campbell, B. 1983, Nature, 303, 584

Hutchings, J. B., Crampton, D., \& Campbell, B. 1984, ApJ, 280, 41

Lim, J \& Ho, P. T. P. 1998, ApJ, 510, L7

Sanders, D. B., Soifer, B. T., Elias, J. H., Madore, F., Matthews, K., Neugebauer, G., \& Scoville, N. Z. 1988a, ApJ, 325, 74

Sanders, D. B., Soifer, B. T., Elias, J. H., Neugebauer, G., \& Matthews, K. 1998b, ApJ, 328, L35 
Smith, E. P., Heckman, T. M., Bothun, G. D., Romanishin, W., \& Balick, B. 1986, ApJ, 306, 64

Véron-Cetty, M. P. \& Véron, P. 1998, ESO Scientific Rep. 18, A Catalog of Quasars and Active Nuclei (8th Ed.; Paris:ESO)

Yee, H. K. C. 1987, AJ, 94, 1461

Yun, M. S., Ho, P. T. P., \& Lo, K. Y. 1996, Nature, 372, 530 